\title{
Two-Dimensional $(r, z)$ Plasma Wave Absorption and Poynting Flux Simulations for Helicon Sources
}

\author{
Y. Mouzouris and J. E. Scharer
}

\begin{abstract}
A two-dimensional $(r, z)$ computer code is utilized to examine wave propagation, collisionless Landau, and collisional heating mechanisms in a helicon source operation.
\end{abstract}

Index Terms-Code, helicon, simulation, waves.

$\mathbf{H}$ ELICON plasma sources are currently of considerable interest for a variety of applications including material surface modification, submicron etching of semiconductors, and for basic plasma source and wave propagation studies. Helicon $(\mathrm{H})$ waves, which are used to create and sustain the plasma, are low frequency $\left(\omega_{c i} \ll \omega \ll \omega_{c e}\right)$ bounded whistler waves [1], [2]. The two propagating modes that are present in the frequency range of interest are the $\mathrm{H}$ mode and the electrostatic Trivelpiece-Gould (TG) mode. The two-dimensional $(r, z)$ plasma wave absorption and electromagnetic Poynting flux flowing from the antenna to the plasma core are examined utilizing graphics capabilities that include contour and surface plots. MAXEB, originally written by Whitson and Berry [3], calculates the electromagnetic wave fields and power absorption in an inhomogeneous cold plasma immersed in a nonuniform magnetic field.

The code has been modified [4] to include an iterative solution that incorporates local thermal effects. The local linear Landau damping term is treated by computing a local $k_{z}$ value using the electromagnetic wave fields previously calculated by the code. These $k_{z}$ values are used to evaluate the kinetic form of the dielectric element $K_{33}$ of the plasma dielectric tensor [1]. The iterative procedure converges rapidly when $\nu / \omega$ is greater than 0.2. At lower gas pressures, $p<1$ mtorr, a convergent solution is reached after 10-15 iterations. A typical run requires about five minutes of run time on a Sun Ultra2 model 2300 workstation.

The modeled cylindrical plasma column [5] is surrounded by a conducting wall of radius $r=8.0 \mathrm{~cm}$. The conducting end plates, as modeled in MAXEB, are located at the axial positions $z=0 \mathrm{~cm}$ and $z=120 \mathrm{~cm}$. A Nagoya type-III antenna of length $l=15 \mathrm{~cm}$ and radius $r=5.1 \mathrm{~cm}$ is centered at $z=27.5 \mathrm{~cm}$. The plasma density profile is assumed to be parabolic in the radial direction $\left[n_{e}(r)=n_{e 0}\left(1-0.9\left(\frac{r}{a}\right)^{2}\right)^{2}\right]$ and uniform in the axial direction. The electron temperature profile is assumed to be uniform throughout the plasma column with an amplitude of $T_{e}=3 \mathrm{eV}$. The gas is argon with a

Manuscript received June 24, 1998; revised October 30, 1998. This work was supported by NSF Grant ECS-9632377.

The authors are with the Department of Electrical and Computer Engineering, University of Wisconsin, Madison, WI 53706 USA (e-mail: scharer@engr.wisc.edu).

Publisher Item Identifier S 0093-3813(99)02598-9.

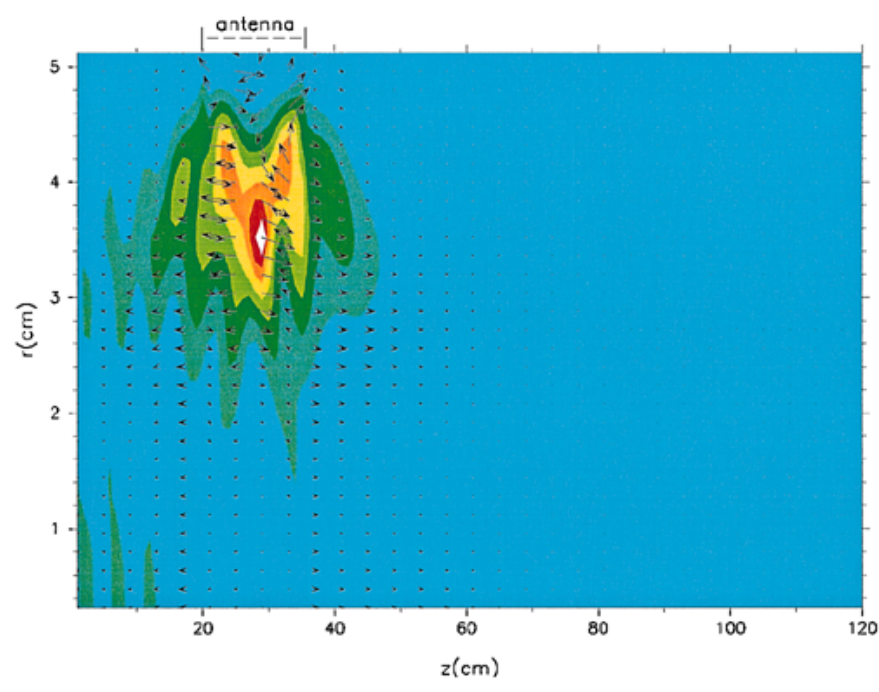

Fig. 1. Power flow and power absorbed by the electrons due to collisional damping of the TG and $\mathrm{H}$ waves for the $m=+1$ mode, $B_{0}=20 \mathrm{G}$, $n_{e 0}=5 \times 10^{11} \mathrm{~cm}^{-3}, f=13.56 \mathrm{MHz}$, and $p=1$ mtorr.

neutral pressure ranging from 1 to 10 mtorr which corresponds to $\nu_{e n} / \omega=0.1-1$ at an electron temperature of $3 \mathrm{eV}$.

Fig. 1 illustrates that at a low magnetic field $\left(B_{0}=20 \mathrm{G}\right)$ and an Ar pressure of 1 mtorr, the absorbed collisional power is localized near the antenna and the coupling is dominated by the TG mode which propagates near the outer plasma edge region. The arrows describe the direction and magnitude of the Poynting vector. The contour plots illustrate the spatial profiles of the absorbed power.

Fig. 2 shows that at higher magnetic fields $\left(B_{0}=110\right.$ $\mathrm{G})$ the coupling is dominated by the helicon mode which transports and deposits the coupled power well away from the antenna region. The TG mode is strongly damped and thus deposits some of the power near the edge of the plasma. The ANTENA2 [6] code is used to illustrate the existence of both modes since MAXEB solves for the total wave field solution and cannot separate the two modes. The contribution of the TG mode to the total absorbed collisional power is significant and dominates the heating process near the surface of the plasma column. In contrast, the $\mathrm{H}$ mode carries and deposits the power through collisional damping away from the antenna region.

Studies using the new, warm plasma, $K_{33}$ term of the dielectric tensor show that collisional damping (by electronneutral collisions) is the dominant heating mechanism for gas pressures greater than 2 mtorr and higher densities $\left(>10^{12}\right.$ $\mathrm{cm}^{-3}$ ). Landau damping on the $3 \mathrm{eV}$ Maxwellian background at a magnetic field $B_{0}=110 \mathrm{G}$ and gas pressure of $5 \mathrm{mtorr}$ is found to be negligible and the power is absorbed entirely 


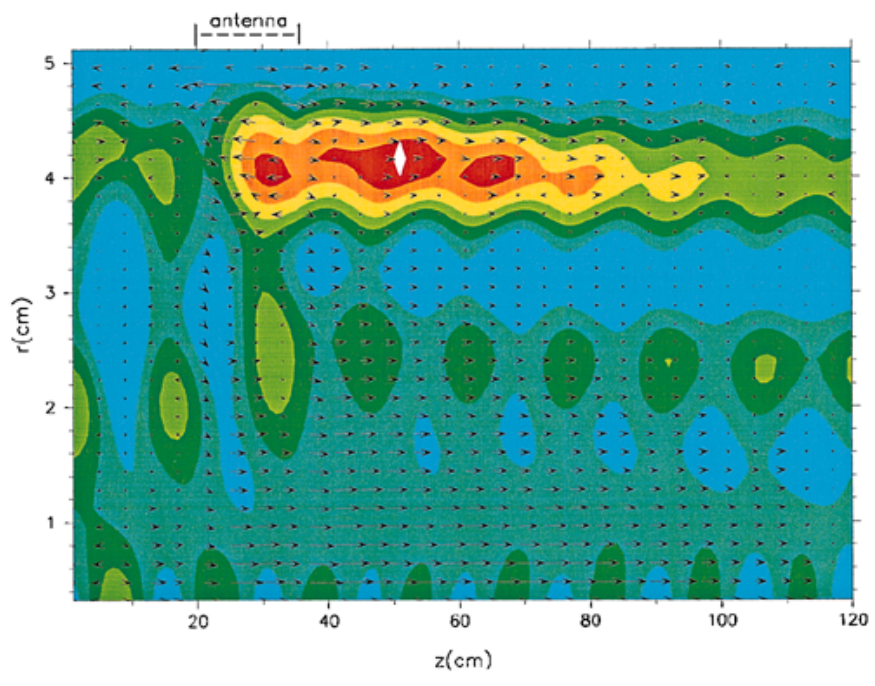

Fig. 2. Power flow and power absorbed by the electrons due to collisional damping of the TG and $\mathrm{H}$ waves for the $m=+1$ mode, $B_{0}=110 \mathrm{G}$, $n_{e 0}=5 \times 10^{11} \mathrm{~cm}^{-3}, f=13.56 \mathrm{MHz}$, and $p=1$ mtorr.

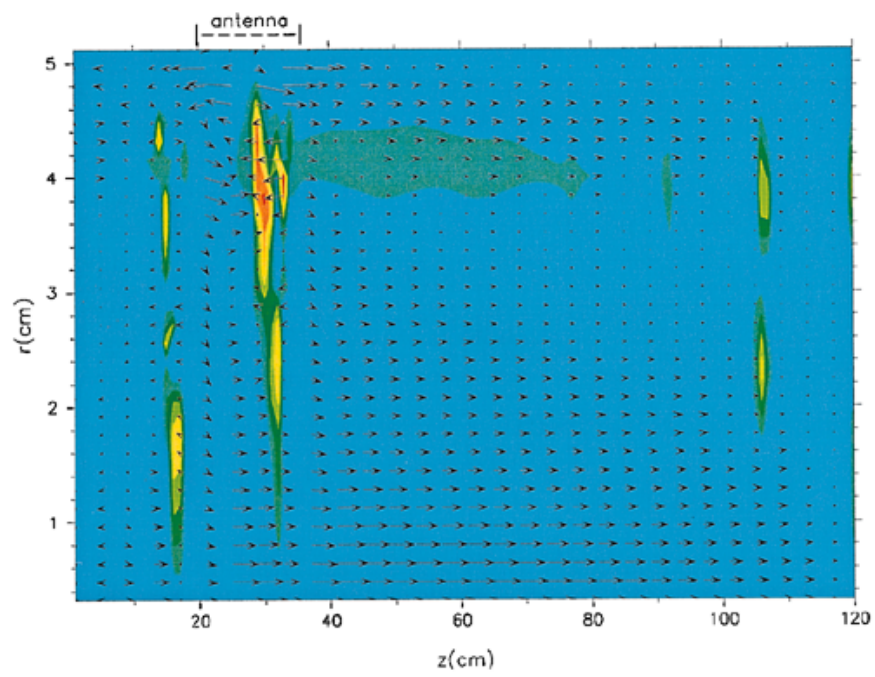

Fig. 3. Power flow and power absorbed by the electrons due to collisionless Landau and collisional damping of the TG and $\mathrm{H}$ waves for the $m=+1$ mode and $B_{0}=110 \mathrm{G}, n_{e 0}=5 \times 10^{11} \mathrm{~cm}^{-3}, f=13.56 \mathrm{MHz}$, and $p=1$ mtorr.

through collisional damping of the wave as it propagates away from the antenna. At lower pressures ( $p=1$ mtorr), as shown in Fig. 3 when compared to Fig. 2, Landau damping becomes important and is incorporated in the calculation of the power absorbed by the electrons. Collisionless Landau damping heats the electrons mainly near the antenna region where the resonant electrons have velocities near the wave phase velocity determined by $v_{\text {ph }}=\omega / k_{z}$.

The experimentally obtained plasma density $\left(n_{e}=10^{13}\right.$ $\left.\mathrm{cm}^{-3}\right)$ and applied magnetic field $\left(B_{0}=800 \mathrm{G}\right)$ profiles [5] are used as input to MAXEB which utilizes the local plasma dielectric tensor for the calculation of the wave fields and the

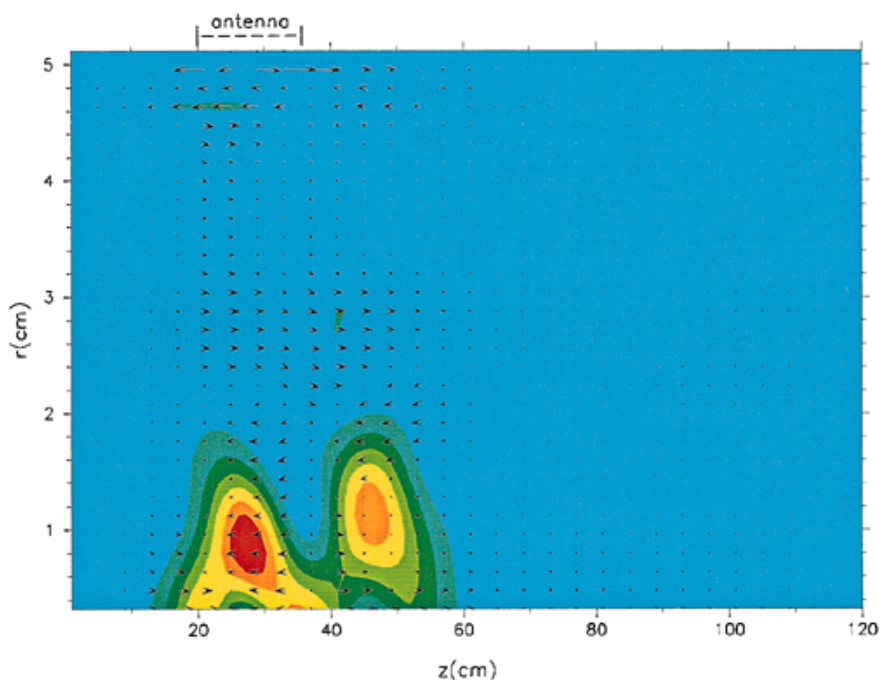

Fig. 4. Power flow and power absorbed by the electrons for the experimentally obtained, axially decaying, plasma density profile for the dominant $m=+1$ mode and $B_{0}=800 \mathrm{G}, f=13.56 \mathrm{MHz}$, and $p=1 \mathrm{mtorr}$.

electron heating profile. The axial plasma density profile is uniform under the antenna and decreases to a negligible value at $z=70 \mathrm{~cm}$. The simulation results were compared with experimental axial wave magnetic field $B_{z}$ data and found to be in good agreement. Fig. 4 illustrates the electron heating profile for a localized wave model utilizing the measured, axially varying plasma density profile. The profile for this case differs considerably from the one computed for an axially uniform plasma which allows the wave to propagate away from the antenna region. The axial inhomogeneities in the plasma density cause distributed reflections and prevent the wave from propagating to the far end of the plasma. The steep density gradient at the axial position $z=70 \mathrm{~cm}$ causes the propagating helicon wave to reflect and thus deposit its wave energy near the antenna core region.

\section{ACKNOWLEDGMENT}

The authors would like to thank L. Berry and J. Whitson for providing a copy of their computer code MAXEB, along with their helpful comments and suggestions. They are also grateful to $\mathrm{M}$. Bettenhausen for many valuable discussions.

\section{REFERENCES}

[1] T. H. Stix, Waves in Plasmas. New York: Amer. Instit. Phys., 1992.

[2] F. F. Chen, "Plasma ionization by helicon waves," Plasma Phys. Control Fusion, vol. 33, no. 11, pp. 339-364, 1991.

[3] L. A. Berry and J. C. Whitson, private communication, 1996.

[4] Y. Mouzouris and J. Scharer, "Wave propagation and absorption simulations for helicon sources," Phys. Plasmas, vol. 5, no. 12, p. 4253, 1998.

[5] X. Guo and J. Scharer, private communication, 1998.

[6] Y. Mouzouris and J. E. Scharer, "Modeling of profile effects for inductive helicon plasma sources," IEEE Trans. Plasma Sci., vol. 24, pp. 152-160, Feb. 1996. 